\title{
Ein Fall von Missbildung, hervorgerufen durch abnorme Engigkeit des Amnion.
}

\author{
Von \\ Dr. Sarwey, \\ Assistenzarzt an der Universitäts-Frauenklinik in Tübingen.
}

(Mit 3 Abbildungen auf Tafel XIII.)

Im Gegensatz zu einem im Bd. 43 dieses Archivs ) von mir beschriebenen Monstrum, welches besonders in geburtshilflicher and pathologisch-anatomischer Beziehung vor anderen ähnlichen Fällen sich auszeichnete, kam im Mai vergangenen Jahres auf der hiesigen Klinik eine Missgeburt zur Beobachtung, welche vorwiegend nach teratologisch-genetischer Richtung hin das grösste Interesse in Anspruch nimmt, sofern die verschiedenen Missbildungen der lebend geborenen Frucht auf ein und dieselbe bestimmte Ursache zurückgeführt werden müssen; schon aus diesem Grunde dürfte die folgende Veröffentlichung, welche ich der gütigen Erlaubniss meines hochverehrten Vorstands, Herrn Professor Dr. v. Säxinger, zu verdanken habe, geboten erscheinen.

\section{Anamnese und Geburtsverlauf.}

Maria R., von Unter-Gröningen, 0.-A. Gaildorf, lediges 21jähriges Bauernmädchen, zum ersten Male schwanger, körperlich und geistig normal entwickelt, war niemals krank gewesen, und bekam die Periode im 16. Lebensjahre, welche von da ab regelmässig alle 4 Wochen, mit mässigem Blutverlust und ohne Schmerzen wiederkehrte. Die letzte Periode trat Ende August ein, die Conceptionszeit verlegte die Schwangere in die erste Woche des September, Kindsbewegungen wurden von ihr nicht wahrgenommen, das Befinden während der Schwangerschaft war stets befriedigend.

Bei der Ende April erfolgten Aufnahme der Schwangeren bietet der

1) Bd. 43,1 . Heft S. $162-180$ 。 
Status praesens keinerlei Besonderheiten; die Beckenmaasse sind: Spin. oss. il. 27, Crist. oss. il. 29, Trochant. 31, Conjug. ext. 19,5, Peripherie des Beckens $90 \mathrm{~cm}$.

Am 15. Mai stellen sich im Verlaufe des Vormittags seltene, kurz dauernde, schon jetzt auffallend schmerzbafte Wehen ein: änsserlich fählt sich der Uterus auch in der Webenpanse gleichmässig gespannt an, wodurch die änssere Untersuchung erheblich erschwert ist; doch lässt sich constatiren, dass die Frucht in Längslage mit nach links gekehrtem Rücken sich befindet, auch sind links in Nabelhöhe deutliche Herztöne zu hören, dagegen keine kleinen Theile und keine Fruchtbewegungen zu sehen oder zu fühlen; bemerkenswerth ist, dass der Kopf der Frucht äusserlich nirgends nachgewiesen werden kann. Die innere Untersuchung liefert folgendes Ergebniss: der äussere Muttermund ist markstückgross, und hat einen derben, scharfen Saum, die noch stehende Blase zieht sowobl in der Wehenpause, als auch während der Wehe glatt und prall gespannt über den änsseren Muttermund hinweg, ohne im Mindesten in denselben hineingetrieben zu werden, und drängt den entfalteten Cervicalkanal halbkuglig nach abwärts (relative Ueberfüllung der Fruchtblase s. u.); hoch über dem Beckeneingang ballotirt ein mit der Fingerspitze eben noch zu erreichender grosser Theil, und dieser letztere wird, obwohl er sich bei der äusseren doppelhänđigen Untersuchung auffallend weich anfühlt, als Kopf angesprochen, da ein solcher an keiner anderen Stelle aufzufinden ist.

In den nächsten 5 Tagen, bis zum 20. Mai, bleibt das Verhalten der Kreissenden genau dasselbe: zeitweise schwache, recht schmerzhaft empfundene Wehen, anhaltende pralle Spannung des unteren Eipols, Ballotement eines grossen Theils über dem Beckeneingang; nur insofern besteht ein Unterschied, als die Eihäute, welche anfangs bis zum Saum des äusseren Vuttermundes mit dem unteren Uterinsegment verklebt waren, von diesem jetzt rings um den Muttermund in ziemlicher Ausdehnung abgelöst sind').

Nachmittags wird die Webenthätigkeit endlich eine bessere, und es folgen bald lrräftige und häufige Wehen, so dass bei dem Abends $10 \frac{1}{2}$ Uhr erfolgenden Blasensprung der Muttermund fünfmarkstück gross ist; der vorliegende Theil ist in den Beckeneingang herabgerückt, und sofort als Steiss zu erkennen: Analöffnung und Labien sind zu touchiren, die Geschlechtslinie verläuft annähernd quer, die Proc. spinos. des Kreuzbeins sind nach links gerichtet, und die Beine an der Vorderfläche des Rumpfes hinaufgeschlagen.

Im Verlaufe der Nacht treiben die Wehen nach völliger Erweiterung des Muttermunds den Steiss bis auf den Beckenboden herab, werden aber gegen Morgen wieder schwächer, weshalb das Beckenende nahezu 1 Stunde lang im Beckenausgang stehen bleibt, (kindliche Herztöne stets gut); erst am 21. Mai Morgens $6 \frac{2}{2} \mathrm{Uhr}$ wird Steiss und untere Rumpfhälfte herausgepresst, und alsbald folgen die Arme und der missbildete Kopf spontan nach: der letztere ist durch eine breite, dünne Membran mit der noch in den Geburtswegen steckenden Placenta in Verbindung, welche indessen sofort nach der Geburt sammt Eihäuten gleichfalls spontan ansgetrieben wird, so dass die genannte Verbindung grösstentheils erhalten bleibt. Die Nachgeburt ist von normaler Grösse,

1) Diese Ablösung war zum Theil spontan erfolgt, zum Theil wurde sie absichtlich mit dem untersuchenden Finger vervollständigt. 
Dicke, Beschaffenheit und $720 \mathrm{~g}$ schwer; die Nabelschnur von Kleinfingerdicke und $18 \mathrm{~cm}$ lang.

Die Wöchnerin macht ein völlig normales Wochenbett durch, und wird 14 Tage post partum entlassen; die missbildete Frucht ist lebend geboren, bewegt Arme und Beine, entleert Meconium, später auch Urin, schreit mit ziemlich lauter Stimme und macht Saugbewegungen; am 2. Tage p. p. nimmat sie einige Male mit dem Löftel eingeflösste Milch begierig zu sich, und stirbt erst am 4. Tage nach der Geburt.

\section{Beschreibung der Missgeburt.}

Die Missbildung stellt eine dem letzten Schwangerschaftsmonat entsprechende Frucht weiblichen Geschlechts dar mit folgenden Körpermaassen:

Gewicht $2500 \mathrm{~g}$; Peripherie des Kopfes (über den Augen) 26,5 cm; Peripherie der Brust in Brustwarzenköhe $31 \mathrm{~cm}$; Länge $44 \mathrm{~cm}$; Peripherie des Hirnsacks $20,5 \mathrm{~cm}$; Peripherie des Bauchs in Nabelhöhe $25,5 \mathrm{~cm}$; Länge der öberen Extremitäten $19 \mathrm{~cm}$; Schulterbreite $13,5 \mathrm{~cm}$; Länge der unteren Extremitäten $20 \mathrm{~cm}$; Hüftenbreite $9,5 \mathrm{~cm}$.

Die Haut des Monstrum ist normal, überall glatt, rosenroth gefärbt, Lanugo findet sich ziemlich reichlich am Rücken, Schultern, Oberarmen und Oberschenkeln, Vernix caseosa spärlicher, in den Inguinalfalten, in der Achselhöhle und am Nabel.

Die Entwicklung von Brust, Bauch und Extremitäten erscheint äusserlich als normal mit Ausnahme zweier Difformitäten, von welchen die eine sich auf die linke Hand bezieht: hier ist nur der Daumen isolirt, die 4 andern Finger sind in einen gemeinsamen Hantsack, wie in einen Fuhrmannshandschuh, eingehüllt, und in diesem leicht gekrümmt, kürzer als an der rechten Hand, aber deutlich von einander isolirt, durchzutasten; die 2 te Anomalie besteht in einem hochgradigen, doppelseitigen, congenitalen Klumpfuss (s. Fig. 1).

Am stärksten verunstaltet ist Schädel und Gesicht: zunächst treten die Bulbi beider Augen stark hervor, eine Stirn als solche fehlt nahezu gänzlich (Krötenkopf); sodann ist eine doppelseitige Lippenspalte 3. Grads vorhanden, welche rechterseits nach oben bis auf $16 \mathrm{~mm}$, linkerseits bis auf $6 \mathrm{~mm}$ an die inneren Augenwinkel hinaufreicht, während die beiden Nasenflügel erheblich in die Breite verzogen sind. Mit der Hasenscharte ist eine doppelseitige Kiefergaumenspalte (Wolfsrachen) combinirt, und an der 
Nasenspitze hängt als rüsselartig hervorstehendes Anhängsel der Zwischenkiefer.

Vom knöchernen Schädel ist nur die Basis vorhanden, die das Schädeldach zusammensetzenden Deckknochen, also Stirnbein bis anf die Pars orbitalis, beide Scheitelbeine, die Schuppentheile der Schläfenbeine und des Hinterhauptbeins, fehlen vollständig; an ihrer Stelle erhebt sich auf der Schädelbasis ein faustgrosser, häutiger, ziemlich schlaffer, weicher, überall glatter, sackartiger Tumor von intensiv blaurother Färbung, welcher sich durch 2 mässig tiefe Furchen in 3 hinter einander liegende, versehieden grosse Lappen scheidet (s. Fig. 2 und 3). Unterhalb dieses Sackes ist die Haut von durchaus normaler Beschaffenheit und mit 3 bis $4 \mathrm{~cm}$ langen braunen Haaren versehen; die Grenze zwischen Haut und Tumoroberfläche wird gebildet durch eine scharf markirte, annähernd horizontal um die Peripherie des Kopfes herumziehende Linie, in welcher die normale Haut direkt und ohne Vermittlung in eine dünne, durchscheinende, gelbliche, mit der Basis des Tumors noch lose und streckenweise verklebte Membran übergeht, die, wie sich aus ihrem ununterbrochenen Zusammenhang mit der Placenta unzweifelhaft ergiebt, nichts anderes ist, als das Amnion. Während die Membran nämlich auf der rechten Seite ir Folge des Geburtsakts in einiger Entfernung von der oben erwähnten Uebergangslinie abgerissen ist, so zwar, dass ein ca. $4 \mathrm{~cm}$ langer, ununterbrochener, und seitlich in die nicht zerrissenen Partien übergehender Rand am Tumor sitzen geblieben ist, bildet sie auf der ganzen linken Hälfte die unmittelbare Fortsetzung des die fötale Fläche der Placenta überziehenden Amnion. Der ganze Tumor ist allseitig vom Amnion umschlossen, nur scheinbar liegt der hinterste, kleinste, nussgrosse Lappen ausserhalb desselben: hier nämlich, entlang der Basis des hintersten Lappens, geht die Epidermis von der behaarten Kopfhaut nicht plötzlich, sondern ganz allmälig sich mehr und mehr verdünnend in den Ueberzug des kleinen Lappens über, und hier ist das Amnion noch mit der Oberfläche desselben in seiner ganzen Ausdehnung so innig verschmolzen, dass es sich von seiner Unterlage ohne Verletzung seiner Continuität nicht lostrennen, sondern erst bei seinem Uebergang vom hinteren kleinsten auf den mittleren grössten Lappen sich mit leichter Mühe als Ganzes vom Tumor abziehen lässt; genau an der genannten Grenze zwischen Haut und Amnion liegt auch der freie, ziemlich scharfe Knochenrand der Schädelbasis. 
Die eigentliche Wand des Tumor wird durch eine $1-2 \mathrm{~mm}$ dicke, glatte, bindegewebige Membran gebildet, deren Innenfläche grösstentheils mit einer schmalen Schicht von Hirnsubstanz innig verbunden ist, stellenweise jedoch glatt über die darunterliegenden Hirntheile hinwegzieht; der Inhalt des Sackes besteht im Wesentlichen aus Gehirnmassen, welche jedoch eine topographisch-anatomische Anordnung der einzelnen Bestandtheile nicht mehr erkennen lassen.

Die Schädelbasis ist überall von der Dura überzogen, und zeigt eine erhebliche Asymmetrie, indem die rechte Schädelgrube $1 \frac{1}{2}$ cm tief, die linke dagegen nur in Gestalt einer seichten Aushöhlung angedeutet ist.

Die inneren Organe der Brust- und Bauchhöhle sind sämmtlich normal entwickelt, der Knochenkern der unteren Epiphyse des Oberschenkels beträgt $5 \mathrm{~mm}$ im Durchmesser.

\section{Beurtheilung.}

An der beschriebenen Missgeburt tritt uns eine Reihe von verschiedenen Abnormitäten entgegen, welche alle mit einer mehr oder weniger bedeutenden Entstellung der äusseren Form einhergehen: Cranioschisis mit Exencephalie, Cheilognathopalatoschisis, beiderseitiger Pes varns, Missbildung der linken Hand. Können oder müssen nun diese sämmtlich auf ein und dieselbe Grundursache zurückgeführt werden, und worin ist diese gemeinsame Ursache zu suchen?

Was die Missbildung an Schädel und Gesicht betrifft, so ist dieselbe, wie aus der vorstehenden Beschreibung und den Abbildungen ohne Weiteres ersichtlich, durch eine Verwachsung der embryonalen Schädelanlage mit der Kopfscheide des Amnion hervorgerufen; ohne auf Einzelheiten einzugehen, 1) sei nur hervorgehoben, dass solche abnorme Verbindungen in Form von Strängen und Bändern als nächste Ursachen der verschiedenartigsten Missbildungen heutzutage allgemein anerkannt sind, und dass die Richtigkeit dieser Erkenntniss gerade durch Beobachtungen,

1) Vgl. Marchand, Missbildungen (Eulenburg'sche Realencyclopädie); Küstner in Müller's Handb. d. Geburtsh., Bd. II, S. 630 ff.; Fromm, Ueber Verwachsungen der menschl. Eihäute mit dem fötal. Schädel 1886; weitere Literaturangaben s. Olshausen, Lehrb. d. Geburtsh., XII. Aufl., S. 503. 
welche, wie die angeführte, die directe Verwachsung zwischen Eihülle und Frucht am anatomischen Präparat noch ad oculos zu demonstriren vermögen, zweifellos erwiesen wird 1). Dass ferner Spaltbildungen, wie Hasenscharte und Wolfsrachen, durch frühzeitige amniotische Verwachsungen oder auch nur durch Einlagerung von Amnionbestandtheilen zwischen die einander entgegenwachsenden Stirn- und Oberkieferfortsätze verursacht werden, ist gleichfalls eine Thatsache, deren allgemeine Anerkennung eine nähere Erörterung überflüssig erscheinen lässt.

Nun entsteht aber weiterhin die Frage, wodurch die amniotische Verwachsung ihrerseits verursacht wurde. Jedenfalls muss dieselbe zu einer Zeit zu Stande gekommen sein, in welcher das eben gebildete Amnion der Embryooberfläche noch innig anlag, in welcher ferner das Medullarrohr noch nicht geschlossen war, und in welcher endlich - will man nicht willkürlich mit älteren Autoren entzündungserregende und geschwürsbildende Momente herbeiziehen - die Epithelzellen der embryonalen Haut noch keine Verhornung zeigten, und da diese drei Voraussetzungen zusammen nur für die allererste Embryonalzeit zutreffen, so muss auch die der Verwachsung zu Grunde liegende Ursache schon in der ersten Schwangerschaftswoche aufgetreten sein.

Normalerweise wird eine Verklebung zwischen Amnion and Embryohaut, deren histologisch-anatomische Gleichwerthigkeit etwaigen Verwachsungsvorgängen an und für sich nur förderlich sein kann, dadurch verhindert, dass das Amnion, sobald es sich über dem Rücken des Foetus zu einem Sack geschlossen hat, theils durch die vermöge seiner Wachsthumsenergie rasch erfolgende weitere Ausdehnung, theils durch die Absonderung von Fruchtwasser alsbald vom Fruchtkörper abgehoben wird; die Möglichkeit einer Verklebung oder Verwachsung ist aber gegeben, sobald diese Berührung zwischen Amnion und Embryo eine länger dauernde wird, und letzteres hinwiederum kann bedingt sein entweder dadurch, dass das Fruchtwasser ungenügend oder zu spät abgesondert wird (Gebr. Braun), oder aber dadurch, dass das Amnion schon in seiner primären Anlage abnorm klein ist, und späterhin der Amnionsack in Folge seiner verminderten Wachsthumsenergie eine für

1) Dem mitgetheilten Falle analoge Beispiele mit Abbildungen s. Marchand a. a. O. (Separatabdruck S. 25); K1otz, Ueber amniotische Bänder und Fäden, Leipzig 1869; Müller, Handb. d. Geburtsh. Bd. II, S. 631 (Buhlscher Fötus) u. A. 
seinen Inhalt verhängnissvoll werdende pathologische Engigkeit besitzt. Die Thatsache, dass unter den gegebenen Verhältnissen amniotische Verwachsungen gerade am Schädel besonders häufig vorkommen, findet nach $\mathrm{Narchand}{ }^{1}$ ) ihre Erklärung darin, dass der Kopftheil am frühesten mit einer Amnionscheide umgeben wird, und daher die Bedingungen für das Zustandekommen der Verwachsung sich gerade an dieser Stelle am häufigsten vorfinden.

Nun liefert, wie ich glaube, der vorliogende Fall eine Bestätigung dafür, dass die soeben genannte, zunächst nur theoretisch als möglich oder wahrscheinlich gedachte letzte Ursache der Verwachsung thatsächlich im Beginn der Eientwicklung vorhanden gewesen ist: Wie aus der Geburtsgeschichte herrorgeht, handelte es sich bei der Kreissenden um eine sogenannte, relative Ueberfüllung der Fruchtblase", eine Anomalie, welche nicht so gar selten vorkommt, und die jedenfalls eine allgemeinere Beachtung verdient, als ihr bis jetzt zu Theil geworden zu sein scheint; wenigstens finde ich dieselbe in den reueren Lehrbüchern der Geburtshilfe (Olshausen, 12. Aufl, Kaltenbach, SpiegelbergWiener, Runge, Martin) nirgends erwähnt, nur in Müller's Handbuch der Geburtshilfe ist sie beiläufig, bei Besprechung der Wehenanomalien vòn J. Veit ${ }^{2}$, gestreift.

Michaelis ${ }^{3}$ ) hat zuerst darauf aufmerksam gemacht, dass es eine Ueberfüllung des Eies giebt, welche, ,auch bei wenigem Wasser statt haben kann, wenn nämlich das Ei verhältnissmässig zu klein ist"; wir sprechen darnach von relativer Ueberfüllung der Fruchtblase dann, wenn am Ende der Schwangerschaft bei normaler Grösse der Frucht, und normaler Quantität des Fruchtwassers die Eihülle, spec. das Amnion, für seinen an und für sich normalen Inhalt zu klein ist - das Wesentliche ist also die abnorme Engigkeit des Amnionsackes, der Inhalt, (Fruchtwassermenge und Eruchtgrösse) wird als innerhalb physiologiseher Grenzen befindlich vorausgesetzt. Natürlicherweise lässt sich die Anwesenheit dieses Zustandes während der Schwangerschaft wohl kaum feststellen, um so leichter aber ergiebt sich im Beginn der Geburt die Diagnose desselben aus folgenden Symptomen: äusserlich ist eine andauernde, gleichmässige Spannung des Fruchthalters auch in

1) a. a. 0.5 .23 .

2) Müller, Bd. II, S. 98.

3) Michaelis, Das enge Becken, Leipzig 1865, S. 184. 
der Wehenpause vorhanden, welche unter Umständen das Heraustasten des Fruchtkörpers beträchtlich erschweren kann, und gleichzeitig besteht primäre Wehenschwäche: Die Wehen sind kurz, selten, wirkungslos und von Anfang an mit einer auffallenden, zu der geringen Intensität der Wehen in keinem Verbältniss stehenden Schmerzhaftigkeit verbunden; innerlich fühlt man während der Wehe, dass der untere Eipol sich nicht, wie gewöhnlich, mehr oder weniger wurstförmig zwischen den wenig erweiterten Muttermund hineindrängt, sondern dass die Eihäute glatt und prall gespannt über den Muttermund hinwegziehen, dass das ganze Ei mehr kugelförmig (Michaelis) bleibt, und in Folge dessen die Erweiterung des Muttermunds nicht zu Stande kommt; dagegen wird das untere Uterinsegment durch die starke Spannung des Eies tief in die Scheide herabgetrieben, wölbt sich wie ein Kugelsegment, welchem zu unterst der pfennig- oder marìstückgrosse Muttermund aufsitzt, nach abwärts, und häufig, wenn auch durchaus nicht immer, bleibt die Ablösung des unteren Eipols rom unteren Uterinsegment aus. Die nachtheiligen Folgen für den Geburtsverlauf sind abnorme Schmerzhaftigkeit, hervorgerufen durch die starke Zerrung des nervenreichen unteren Uterinsegments, mangelhafte Erweiterung der weichen Geburtswege, Verzögerung, bez. Stillstand der Geburt mit beweglichem Hochstand des vorliegenden Theils.

Die Therapie besteht einfach darin, dass man mit dem untersuchenden Finger den unteren Fipol in möglichst grosser Ausdehnung vom unteren Uterinsegment Ioslöst, vorsichtig die Blase sprengt, und das Fruchtwasser möglichst langsam abfliessen lässt'). Wie Michaelis ${ }^{2}$ ) betont, ist in solchen Fällen dieses Verfahren ausserordentlich wirkungsvoll, denn Geburten, welche sich bis dahin Tage lang verzögert hatten, erfolgten nicht selten innerhalb 1 Stunde, ja noch schneller.

Diese relative Ueberfüllung der Fruchtblase kann nun aber nicht erst in einer späteren Zeit der Schwangerschaft plötzlich und unvermittelt entstanden sein, sondern sie muss nach meinem Dafürhalten zurüekgeführt werden auf eine Bildungsanomalie des Amnion, welche in einer abnormen Kleinheit der Amnion-

1) Im vorliegenden Fall wurde auf den Blasensprung verzichtet, weil eine sichere Diagnose des rorliegenden Theils nicht gestellt war.

2) Michaelis a. a. O. 
anlage besteht, und somit gestattet die im Beginn der Geburt constatirte relative Ueberfüllung der Blase den Rückschluss, dass eine allgemeine Engigkeit des Amnion schon vom ersten Beginn der Gravidität an bestanden haben muss. Diese hat aber im vorliegenden Falle nicht nur zu der Verwachsung und Missbildung am Kopf Veranlassung gegeben, sondern es hat auch die durch die ungenügende Bildung des Amnion entstandene Raumbeschränkung zu der Klampfussstellung und zu der Einhüllung der Finger in einen gemeinsamen Hautüberzug geführt' ${ }^{1}$ ).

Selbstverständlich wird mit einer relativen Ueberfüllung der Blase keineswegs immer eine fötale Formanomalie nothwendig verbunden sein müssen, doch erscheint es nicht unwahrscheinlich, dass in ähnlichen Fällen von Missbildungen die relative Ueberfüllung der Blase als Folgezustand der abnormen Kleinheit der Amnionanlage bei genauer Beobachtung häufiger angetroffen werden kann.

Es handelt sich somit in der vorliegenden Beobachtung um einen Fall, in welchem sämmtliche äusseren Formanomalien auf dieselbe Grundursache zurückzuführen sind, nämlich auf die abnorm enge Beschaffenheit des Amnionsackes, welche durch die ganze Zeit der Schwangerschaft hindurch rom Beginne an vorhanden war, und welche in frühester Zeit der Entwicklung theils mit Verwachsung, theils ohne eine solche lediglich durch die damit verbundene Raumbeschränkung die genannten, verschiedenartigen Abnormitäten hervorgerufen hat.

1) Vgl. Marchand a. a. 0. S. 28. 